\title{
A case of cytokine hemofiltration and extracorporeal life support as treatment of coronavirus disease 2019 (COVID-19) respiratory failure
}

\author{
Omar Al-Qudsi, MD, ${ }^{\mathrm{a}}$ Bryan Whitson, MD, PhD, ${ }^{\mathrm{b}}$ Amar Bhatt, MD, ${ }^{\mathrm{a}}$ Sheila Chucta, DNP, CNS, ${ }^{\mathrm{c}}$ and \\ Ravi Tripathi, MD, MBA, ${ }^{\text {a }}$ Columbus, Ohio
}

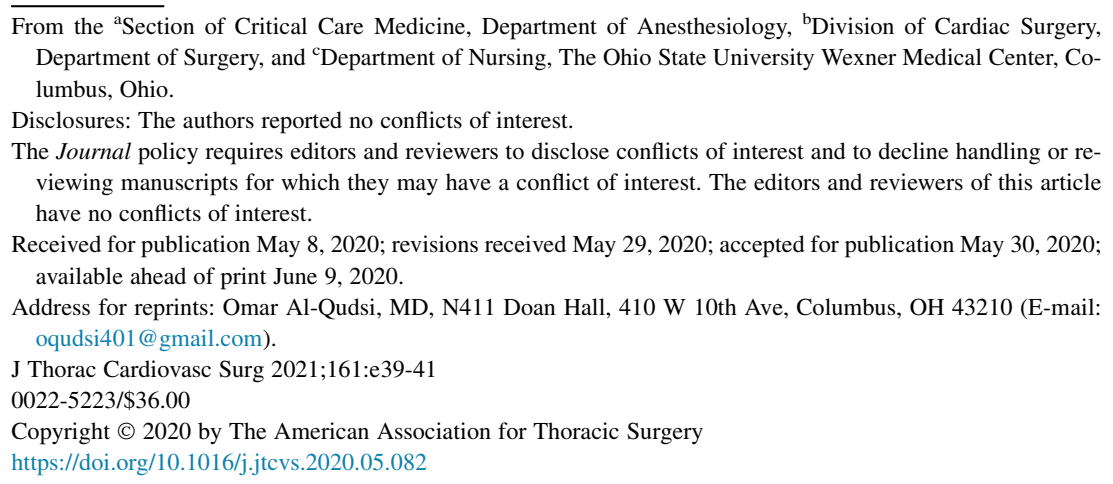

Despite the critical role of cytokines in the modulation of the immune response, cytokine hyperactivity has been implicated in the pathogenesis of severe organ failure secondary to sepsis, cardiac surgery, immunotherapy, and a myriad of other disease states. ${ }^{1-3}$ Mitigation of inflammatory overactivity is a therapeutic option that has garnered much interest over the years. In addition to corticosteroids and immunoglobulins, cytokine hemofiltration can be another such option, helping to attenuate plasma levels of inflammatory markers.

As of this time, the exact pathogenesis of coronavirus disease 2019 (COVID-19) is unknown, but a hyperactive immune response has been implicated as a possible cause of the ensuing, overwhelming cytokine storm. ${ }^{4}$ Cytokine hemofiltration has been endorsed as a treatment therapy for patients with acute kidney injury requiring renalreplacement therapy in some COVID-19 epicenters. ${ }^{5}$ The fundamental difficulty remains in selecting patients with a hyperactive immune response and determining the optimal timing for therapy. Trending of inflammatory markers is one such stratification method, but given the low risk associated with hemofiltration, use in refractory hypoxemia should be considered.

\section{CASE REPORT}

We report a 53-year-old female health care worker with a medical history only significant for obesity (body mass index of 38). She presented to the emergency department after 2 days of symptoms following a recent exposure to a patient who was positive for COVID-19. Admission pulseoximeter saturation was $93 \%$ on $2 \mathrm{~L}$ of nasal cannula oxygen with evidence of bilateral infiltrates on chest radiograph

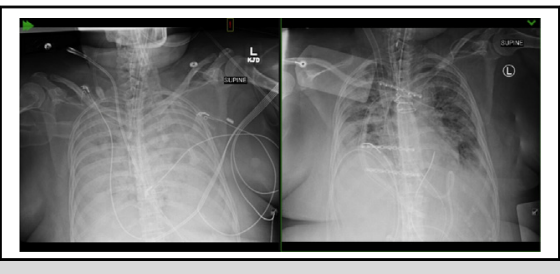

Radiographic improvement following treatment with cytokine hemofiltration.

CENTRAL MESSAGE

A hyperactive immune response has been implicated in COVID-19 respiratory failure. Hemofiltration with a membrane designed to remove cytokines offers a potential avenue for treatment.

See Commentary on page e43.

and a positive rapid COVID-19 screening test. Initial therapy consisted of hydroxychloroquine and empiric broadspectrum antibiosis. Progressively, her oxygen requirement rapidly escalated, culminating in intubation and ventilatory requirement of 1.0 inspired oxygen fraction $\left(\mathrm{FIO}_{2}\right)$ and 16 $\mathrm{cm} \mathrm{H}_{2} \mathrm{O}$ of positive end-expiratory pressure on hospital day 2. Adjunctive therapies for refractory hypoxemia over the next few days included prone positioning, dexamethasone, intravenous diuretics, as well as experimental therapy with the monoclonal interleukin (IL)-6 antibody, tocilizumab. Despite aggressive treatment, her IL-6 levels hovered around 600 to $875 \mathrm{pg} / \mathrm{mL}$ and D-dimer remained mildly elevated. As her oxygenation continued to deteriorate, she did receive experimental convalescent plasma therapy with no improvement. As her oxygenation and lung compliance continued to worsen despite optimal medical therapy, she was placed on veno-venous extracorporeal life support (VV-ECLS) in accordance with institutional guidelines.

She was uneventfully cannulated on day 7 of mechanical ventilation by using a femoral-femoral configuration $(25-\mathrm{F}$ multistage drainage cannula and 23-F single-stage return cannula), which was subsequently converted to a bi-caval, 27-F dual-lumen cannula via the right internal jugular 
vein to assist with mobility. Following initiation of VVECLS, her ventilator settings were able to be brought down into a more lung protective range. With ECLS blood flow of $\sim 3.5 \mathrm{~L}$ per minute and $1.0 \mathrm{FIO}_{2}$ through the circuit, her arterial oxygen tension: $\mathrm{FiO}_{2}$ ratio remained 74 to $100 \mathrm{~mm} \mathrm{Hg}$ and her lung compliance was estimated at $3.8 \mathrm{~mL} / \mathrm{cm} \mathrm{H}_{2} \mathrm{O}$. Along with her pulmonary parameters, her inflammatory markers continued to increase, with IL6 levels measured at $2242 \mathrm{pg} / \mathrm{mL}$ and tumor necrosis factor alpha (TNF- $\alpha$ ) 7.7 (ref range $<4.0 \mathrm{pg} / \mathrm{mL}$ ). Due to her worsening clinical status, the decision was made to proceed with cytokine hemofiltration. Access for hemofiltration was obtained with a standard 13-French dual-lumen hemodialysis catheter. Her treatment regimen consisted of 2 sessions on consecutive days, each lasting 12 hours. The treatments were performed using a SeaStar CLR 2.0 (SeaStar Medical, Denver, Colo) high cut-off filter and continuous venovenous hemofiltration using a clearance therapy of $35 \mathrm{~mL} / \mathrm{kg} / \mathrm{h}$ and a filtration fraction of $<0.1$. Within 12 hours of each treatment, both IL- 6 levels and TNF- $\alpha$ dropped by a magnitude of $25 \%$.

In the 36 hours following cytokine filtration, compliance was noted to increase 3 -fold $\left(10.4 \mathrm{~cm} / \mathrm{mL} \mathrm{H}_{2} \mathrm{O}\right)$ and her arterial oxygen tension: $\mathrm{Fio}_{2}$ ratio rose to 240 (Table 1). Her chest radiograph also demonstrated a remarkable progression, with near-complete resolution of her bilateral opacities and air bronchograms (Figure 1). She was subsequently weaned and decannulated from VV-ECLS over the next 4 days, and tracheostomy was performed to assist with liberation from mechanical ventilation. Her tracheostomy has since been reversed, and she has been discharged to rehabilitation. Of note, she continues to test positive for COVID-19 despite convalescent plasma and confirmed immunoglobulin $\mathrm{G}$ antibodies. Institutional review board approval was waived due to the single case report nature. Consent was obtained for the use of images and case data.

\section{DISCUSSION}

The variable presentation and rapid clinical decompensation of patients with COVID-19 have been attributed to a variety of pathologic conditions, of which cytokine release syndrome has been implicated, leading to the most severe forms of acute respiratory distress syndrome (ARDS) and multisystem organ failure. ${ }^{1,4,6}$ To our knowledge, this is the first report of using cytokine hemofiltration for the successful treatment of COVID-19-related severe ARDS with concomitant VV-ECLS.

Cytokines are inflammatory mediators with molecular weights ranging from $\sim 5$ to $20 \mathrm{kDa}$, the most notable of which are IL-6, IL-8, and TNF- $\alpha$. They play an important role in cell signaling as endogenous pyrogens, stimulators of systemic inflammation, and up-regulators of other acute-phase proteins and cells. Although their role in systemic inflammation is well known, the etiology in COVID-19 is still poorly understood. ${ }^{7}$ Limited research evolving from Wuhan, China, indicates that a secondary hemophagocytic lymphohistiocytosis-like syndrome could be at the center of the cytokine release syndrome (CRS) exhibited by the COVID-19 infection. The syndrome is characterized by elevated levels of IL-2, IL-6, ferritin, and IL-7 among other mediator, although a multicenter review found that IL- 6 and ferritin levels were significant predictors of mortality. ${ }^{4}$ In CRS, elevated levels of ILs result in widespread activation of cell-signaling, with secretion of vascular endothelial growth factor and reduced E-cadherin expression contributing to increased vascular permeability and leakage. ${ }^{8}$ Given the role of CRS in COVID-19 and its downstream effects on multisystem organ failure and severe ARDS, mitigation of cytokines may prove to be an effective treatment modality.

Cytokine filtration using a highly porous bead membrane has previously been advocated in attenuating a prolonged harmful host response, with applications extending from sepsis to cardiac surgery. ${ }^{9}$ In addition, hemoadsorption therapy has been advocated for specific use in ECLS to control the proinflammatory and complement activation by extracorporeal membranes. ${ }^{10}$ Global immunosuppressive effects are limited by the cutoff of the filter of approximately $50 \mathrm{kDa}$, as most immunoglobulins are approximately $100 \mathrm{kDa}$ in size. The SeaStar CLR 2.0 is one such device, using a polysulfone membrane with a larger cut-off for protein permeability to help adsorb circulating cytokines. Our decision to treat this patient with cytokine hemofiltration was based on her worsening clinical status with concomitantly elevated inflammatory markers while keeping in

TABLE 1. Changes in parameters of oxygenation that correspond to treatment with a cytokine hemofilter while on VV-ECLS

\begin{tabular}{|c|c|c|c|c|c|}
\hline & ECLS day 1 & ECLS day 2 (treatment 1) & ECLS day 3 (treatment 2) & ECLS day 4 & ECLS day 5 \\
\hline $\mathrm{PaO}_{2}: \mathrm{FIO}_{2}$ ratio & 74 & 99 & 89 & 174 & 240 \\
\hline Ventilator $\mathrm{FIO}_{2}$ & 1.0 & 1.0 & 1.0 & 0.9 & 0.4 \\
\hline Compliance, $\mathrm{mL} / \mathrm{cmH}_{2} \mathrm{O}$ & 3.8 & 5.0 & 4.7 & 9.3 & 10.4 \\
\hline ECLS sweep, LPM & 5 & 7 & 5 & 4 & 1 \\
\hline Daily fluid balance, $\mathrm{mL}$ & 930 & -260 & 990 & 2130 & -540 \\
\hline
\end{tabular}

ECLS, Extracorporeal life support; $\mathrm{PaO}_{2}$, arterial oxygen tension; $\mathrm{FIO}_{2}$, inspired oxygen fraction; $\mathrm{LPM}$, liters per minute. 


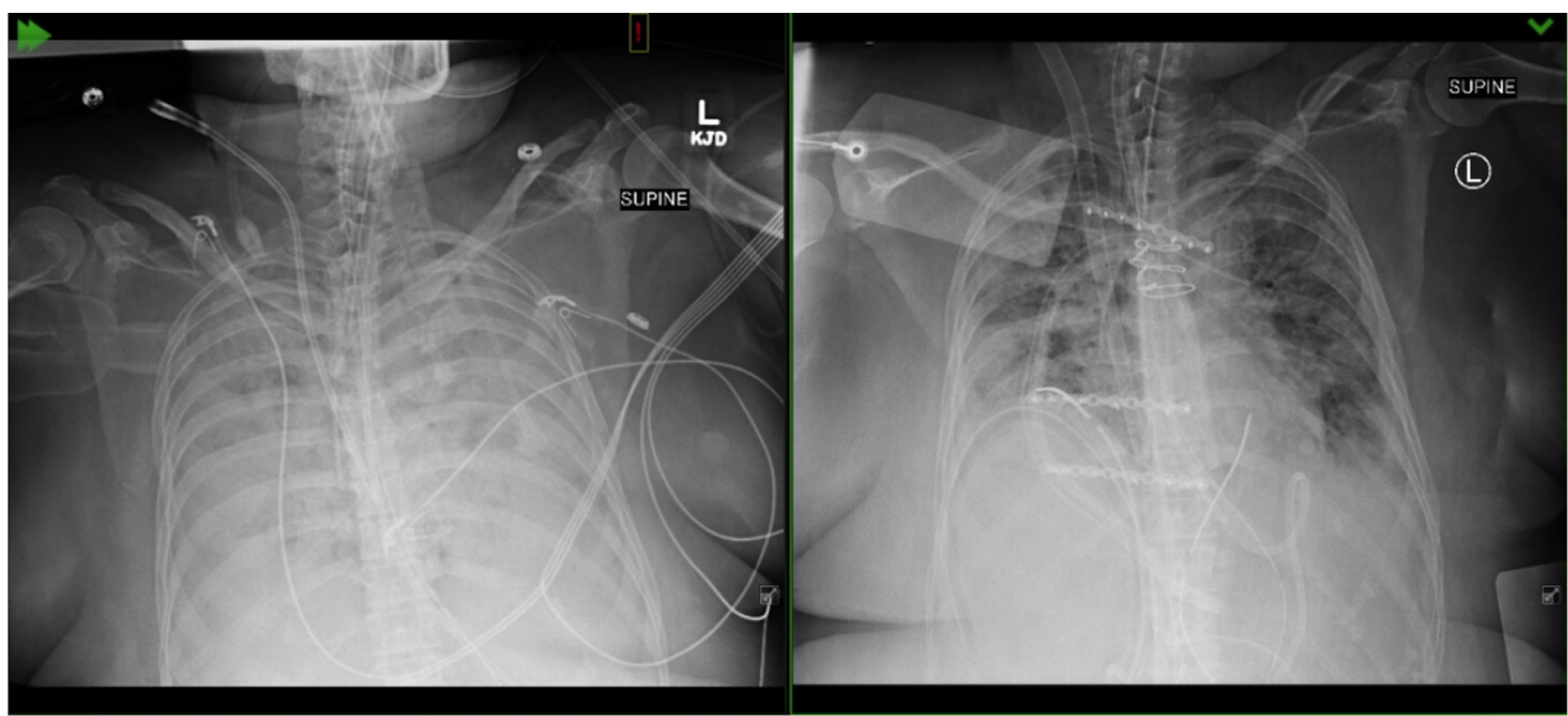

FIGURE 1. Left, Radiograph revealing bilateral consolidation and air bronchograms due to coronavirus respiratory failure before treatment with a cytokine hemofilter. Right, Radiograph obtained $\sim 36$ hours following treatment demonstrating improved aeration bilaterally.

mind the relatively safe side-effect profile of treatment. Given the numerous treatment modalities that our patient received, we cannot conclude with certainty that cytokine filtration was the only mechanism for clinical improvement. However, the linear correlation between inflammatory marker decline and improvement in oxygenation supports our hypothesis. Furthermore, the persistently positive novel-severe acute respiratory syndrome coronavirus 2 respiratory swabs despite administration of convalescent plasma lends credence to the idea that a hyperactive immune system may be responsible for the ongoing pathogenesis associated with COVID-19 as opposed to simple viremia.

Ongoing research involving a larger number of patients, preferably in a randomized controlled fashion, is needed to accurately risk-stratify patients and continue to tailor therapy. Our case report highlights the novel use of cytokine hemofiltration for treatment of severe ARDS secondary to COVID-19 in a patient on VV-ECLS.

\section{References}

1. Shimabukuro-Vornhagen A, Gödel P, Subklewe M, Stemmler HJ, Schlößer HA, Schlaak M, et al. Cytokine release syndrome. J Immunother Cancer. 2018;6:56.

2. Chaudhry H, Zhou J, Zhong Y, Mustafa Ali M, McGuire F, Nagarkatti PS, et al. Role of cytokines as a double-edged sword in sepsis. In Vivo. 2013;27:669-84.

3. Tonnesen E, Christensen VB, Toft P. The role of cytokines in cardiac surgery. Int J Cardiol. 1996;53(suppl):S1-10.

4. Mehta P, McAuley DF, Brown M, Sanchez E, Tattersall RS, Manson JJ, et al. COVID-19: consider cytokine storm syndromes and immunosuppression. Lancet. 2020;395:1033-4.

5. Alberici F, Delbarba E, Manenti C, Econimo L, Valerio F, Pola A, et al. Management of patients on dialysis and with kidney transplant during SARS-COV-2 (COVID-19) pandemic in Brescia, Italy. Kidney Int Rep. 2020;5:580-5.

6. Pedersen SF, Ho YC. SARS-CoV-2: a storm is raging. J Clin Invest. 2020;130: 2202-5.

7. Liu B, Li M, Zhou Z, Guan X, Xiang Y. Can we use interleukin-6 (IL-6) blockade for coronavirus disease 2019 (COVID-19)-induced cytokine release syndrome (CRS)? J Autoimmun. 2020;111:102452.

8. Moore J, June CH. Cytokine release syndrome in severe COVID-19. Science. 2020;368:473-4

9. Friesecke S, Stecher SS, Gross S, Felix SB, Nierhaus A. Extracorporeal cytokine elimination as rescue therapy in refractory septic shock: a prospective single-center study. J Artif Organs. 2017;20:252-9.

10. Datzmen T, Träger K. Extracorporeal membrane oxygenation and cytokine adsorption. J Thorac Dis. 2018;10(suppl 5):S653-60. 